\title{
TREINO DE FORÇA: UMA REVISÃO SISTEMÁTICA SOBRE O VOLUME DE EXERCÍCIOS UTILIZADOS PARA EMAGRECIMENTO
}

\author{
José Nunes da Silva Filho ${ }^{1}$, Robson Alex Ferreira ${ }^{2}$ \\ ${ }^{1}$ Universidade Gama Filho, Programa de Pós-Graduação em Ciências do Exercício e do Esporte, Rio de Janeiro, RJ. \\ ${ }^{2}$ Universidade do Estado do Matogrosso - UNEMAT, Cáceres, MT. e-mail: jose nunes99@hotmail.com
}

\section{RESUMO}

O sobrepeso e a obesidade são considerados, juntos, a principal causa de doenças sistêmicas, representando o quinto risco de mortes globais e provocando a morte de certa de 2,8 milhões de adultos ao ano. $O$ treino de força vem sendo propostos como auxílio na prevenção e tratamento da obesidade. Portanto, o objetivo do estudo foi verificar as evidências sobre o número de exercícios utilizados no treino de força quando voltado para o emagrecimento. Trata-se de uma revisão sistemática, descrita seguindo as recomendações PRISMA. A escolha dos termos chaves e seus sinônimos aconteceram nos descritores de saúde no Decs e no Mesh. Foram selecionados os termos chave na língua portuguesa: Treinamento de força; Treinamento Resistido; Número de Exercícios; Perda de Peso e os equivalentes na língua inglesa. Após, foi realizada busca nas principais bases de dados: SciELO, PUBMED e no site de busca Scholar Google. O levantamento de dados foi realizando no período de dois meses entre Dez/2013 a Jan/2014. Foram contemplados para pesquisa 15 estudos, e o número mínimo e máximo de exercícios que utilizaram nos experimentos foram entre 6 a 12 exercícios respectivamente, notou-se também que 14 (93,3\%) dos estudos obtiveram respostas significativas na redução da porcentagem de gordura. Portanto, conclui-se que o número de exercícios utilizados nos ensaios contemplados esteve em torno de 6 a 12 exercícios e, quando ajustados obtiveram um número médio de $8,8 \pm 1,9$ exercícios.

Palavras-chave: treino de força, treinamento resistido, número de exercícios, variáveis do treinamento, perda de peso.

\section{STRENGTH TRAINING: A SYSTEMATIC REVIEW ON THE VOLUME OF EXERCISE USED FOR WEIGHT LOSS}

\begin{abstract}
Overweight and obesity are considered, together, the leading cause of systemic diseases, representing the fifth overall risk of death, by provoking death of some 2.8 million adults per year. Strength training has been proposed as an aid in the prevention and treatment of obesity. Therefore, the purpose of the study was to investigate the evidence on the number of exercises used in strength training when facing the weight loss. This is a systematic review, described following the PRISMA recommendations. The choice of key terms and their synonyms happened in health descriptors in DeSC and MeSH. We selected the key terms, Resistance Training, Strength Training, Number of exercise, Weight Loss and the equivalents on Portuguese. After, it was take a search in the main databases: SciELO, PUBMED and Google Scholar search site. The survey was performing during the two months between the Dec/2013 Jan/2014. Were awarded to 15 research studies, and the minimum and maximum number of exercises used in the experiments were between 6 to 12 exercise respectively, it was noted also that 14 (93.3\%) of the studies reported significant responses in reducing the percentage of fat. Therefore it is concluded that the
\end{abstract}


number of exercises used in the included trials was around 6 to 12 exercise, and when adjusted earned an average number $8.8 \pm 1.9$ exercises.

Keywords: strength training, resistance training; number of exercise, variables training, weight loss. 


\section{INTRODUÇÃO}

$O$ treino de força (TF) segundo a American College of Sport Medicine (ACSM) ${ }^{1}$ pode ser descrito pela execução de exercícios que realizam contrações voluntárias utilizando a musculatura esquelética uma resistência, podendo ser através de pesos livres, máquinas ou até mesmo com o próprio corpo; caracterizados por exercícios dinâmicos (isotônicos ou isocinéticos), ou exercícios estáticos (isométricos). O TF também pode ser encontrado descrito na literatura como treino resistido, devido seu princípio estar correlacionado com atuação da musculatura contra certa resistência ${ }^{2}$. Atualmente o TF vem crescendo de forma considerável por demonstrar-se como mediador incontestável para saúde, sendo sugerido tanto na prevenção quanto no tratamento de inúmeras doenças sistêmicas ${ }^{3}$.

Segundo a World Health Organization ${ }^{4}$, atualmente, o excesso de peso, vem sendo um dos principais problemas da população mundial, pois, o sobrepeso e a obesidade juntos são a principal causa de doenças sistêmicas, representando $05^{\circ}$ risco de mortes globais e provocando a morte de cerca de 2,8 milhões de adultos ao ano. Para descrever o estado de sobrepeso e obesidade, os estudos epidemiológicos utilizam o índice de massa corpora (IMC), calculado a partir da formula $\left(\mathrm{IMC}=\mathrm{Kg} / \mathrm{m}^{2}\right)^{5}$.
A obesidade vem sendo considerada sério problema de saúde publica mundial, portanto, buscam-se alternativas para prevenção e/ou tratamento desta doença. Sendo a redução do peso corporal é um dos tratamentos eficazes mais comuns na perda de peso $^{6}$, podendo ocorrer através da redução da ingestão calórica, ou até mesmo, pelo aumento do gasto calórico, este ultimo que pode ser potencializado consideravelmente através da pratica atividade física ${ }^{7,8}$.

Como o exercício físico já esta consolidado na literatura por ser um meio eficiente na prevenção e tratamento da obesidade, diversos tipos de exercícios vem sendo proposto para finalidade, exercícios de caminhada $^{9-11}$, exercícios aeróbios ${ }^{12}$, exercícios anaeróbios ${ }^{13}$, ou até mesmo a combinação entre exercícios aeróbios e anaeróbios $^{14}$. Acredita-se que 0 gasto calórico eleva-se com a prática de exercícios físicos através do aumento na taxa de metabolismo basal (TMB), este que é considerado o mais importante componente do gasto energético diário no indivíduo ${ }^{15}$.

Ao correlacionar a prática de exercícios físicos com a elevação da TMB, o TF vem mostrando-se bastante eficaz tanto em respostas agudas ${ }^{16}$, quanto crônicas, até mesmo quando comparados aos exercícios aeróbios ${ }^{17}$. Segundo Silva Filho $^{3}$ essas 
respostas podem ser explicadas tendo em vista que o TF provoca simultaneamente perda de massa gorda e preservando ou aumentando a massa magra, o que contribui significativamente para o aumento da TMB e, por conseguinte, melhoras na composição corporal total.

Os benefícios do treino de força para o emagrecimento já estão bem consolidados na literatura, entretanto, para que esses benefícios ocorram de forma harmoniosa com a saúde dos indivíduos, há necessidade de manipulação correta de vários fatores, conceituados como variáveis do treinamento, sendo o volume e intensidade as principais variáveis $^{18,19}$. Estas importantes variáveis são completadas por outras: número de exercícios, número de séries, número de repetições, intervalos, velocidade de execução, sobrecarga etc ${ }^{20,21}$. Porém para Silva e Farinatti ${ }^{22}$, a manipulação das variáveis no TF para alguns objetivos, não esta completamente descrita na literatura.

Dentre as subvariáveis do treinamento, o número ideal de exercícios a ser utilizado no TF objetivando emagrecimento, ainda não apresenta consenso na literatura, apesar fator importantíssimo na prescrição dos exercícios, tendo em vista que a manipulação demasiada deste leva o indivíduo ao chamado overtraining, trazendo sérios problemas para saúde do praticante ${ }^{23}$.
O objetivo desse estudo foi verificar evidências científicas sobre uma das variáveis do treinamento, especificamente o número de exercícios utilizados em experimentos clínicos sobre os efeitos do TF voltados para o emagrecimento.

\section{MATERIAIS E MÉTODO}

A presente pesquisa enquadra-se como sendo uma revisão sistemática, por angariar respostas na literatura de forma não tendenciosa ${ }^{24}$, e com intuito apresentar de forma técnica a redação dos dados, optou-se por seguir as recomendações para do Preferred reporting items for Systematic Reviews and Meta-análise - PRISMA ${ }^{25}$. A busca de informações foi realizada nas bases de dados nacionais e internacionais: SciELO, PubMed e no site de busca Google Acadêmico, por haver um grande número de revistas que não estão disponíveis nas bases supracitadas.

\section{BUSCA DOS ARTIGOS}

Previamente à busca dos estudos, houve a definição de termos chave, seguindo os Descritores em Saúde DeSC (http://decs.bvs.br) para bases de dados nacionais e $\mathrm{MeSH}$ (http://www.ncbi.nlm.nih.gov/mesh/) para as internacionais. Forma definidos os termos: Treinamento de força; Musculação; Treinamento Resistido; Número de 
Exercícios; Perda de Peso. Na língua inglesa: Resistance Training; Strength Training; Muscle Strength; Number of exercise; Weight Loss. O levantamento de dados foi realizando entre os meses de Dez/2013 a Jan/2014.

\section{CRITÉRIOS DE INCLUSÃO DE ARTIGOS}

Foram selecionados apenas os experimentos clínicos que utilizaram o treinamento resistido como variável independente e o emagrecimento como variável dependente. Os estudos foram selecionados também, mesmo com a presença de outra variável independente, e impreterivelmente, os ensaios deveriam descrever o número de exercícios utilizados no tratamento. Não foram incluídos trabalhos de conclusão de curso (monografias, dissertações ou teses), ou trabalhos que utilizaram experimentos com animais.

\section{CRITÉRIOS DE ELEGIBILIDADE}

A primeira seleção dos artigos aconteceu pela leitura do título, seguindo da leitura do resumo, e caso atendesse aos critérios de inclusão, optava-se pela leitura completa do estudo, para que fosse verificado na integra os critérios de elegibilidade e somente após estas etapas considerar o estudo contemplado. A seleção dos estudos esta devidamente representada na Figura 1.

\section{ANÁLISE ESTATÍSTICA}

Depois de realizada a seleção dos estudos e observação dos dados, foi utilizado o software IBM ${ }^{\circledR}$ SPSS $^{\circledR}$ Statistic, vs 21 (Nova York/EUA, 2012), verificando através de estatística descritiva (média e desvios padrão) do número de exercícios prescritos nos estudos contemplados.

\section{RESULTADOS}

Na Tabela 1 são apresentados os 15 estudos contemplados. Encontrou-se 08 publicações nacionais e 07 internacionais que apresentavam o número de exercícios utilizados no treino de força voltado para o emagrecimento. Os números mínimos e máximos dos exercícios encontrados ficaram entre 06 a 12 exercícios. 


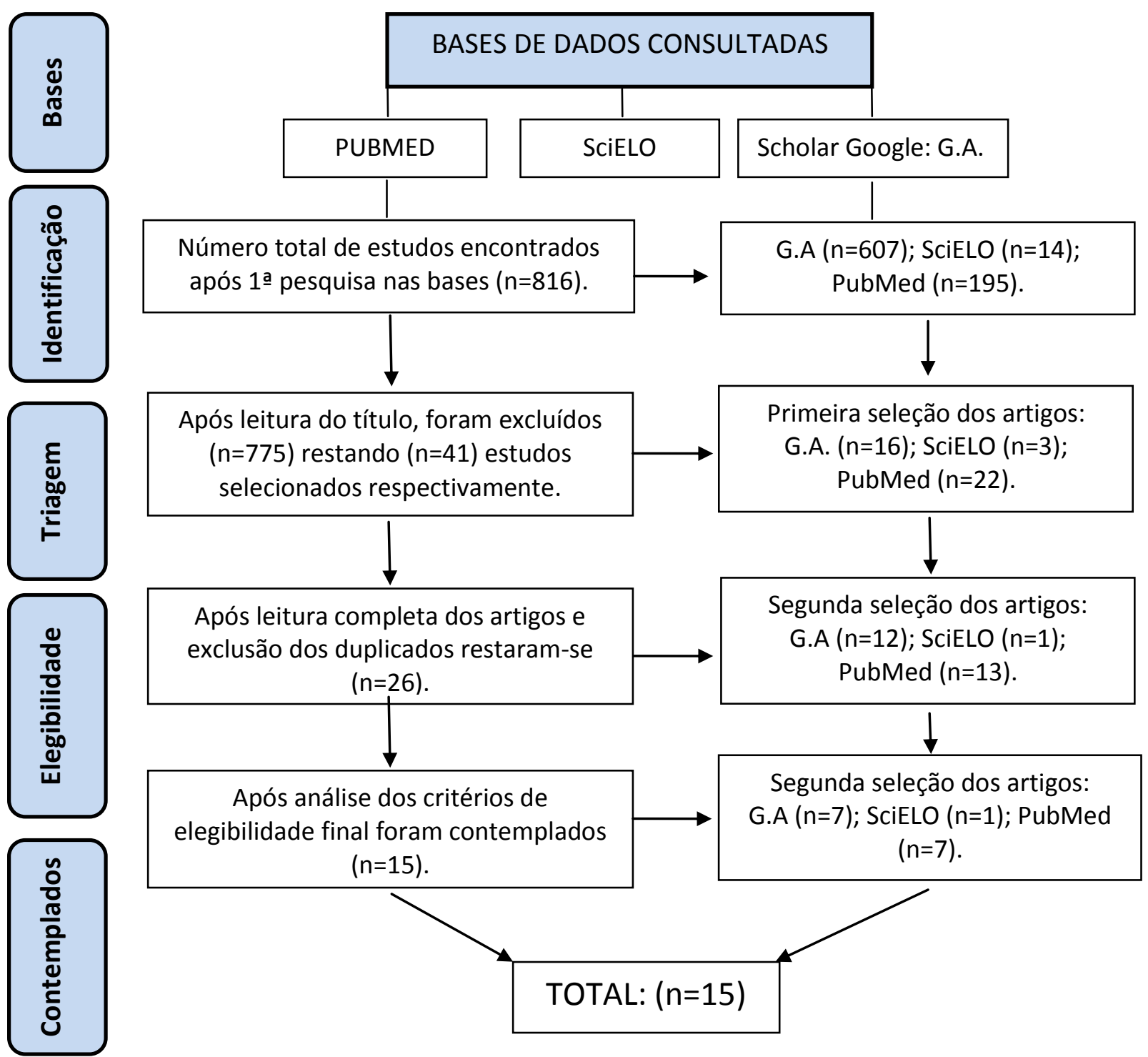

Figura 1. Fluxograma da seleção dos estudos.

Tabela 1. Detalhes dos estudos contemplados.

\begin{tabular}{cccc}
\hline $\mathbf{N}^{\circ}$ & Estudo & Ano & Desfecho \\
\hline 1 & Kraemer et al. $^{26}$ & 1997 & $<\% \mathrm{G}$ \\
\hline 2 & Prabhakaran et al. $^{13}$ & 1999 & $<\% \mathrm{G}$ \\
\hline 3 & Dos Santos et al. $^{27}$ & 2002 & $\tilde{\mathrm{n}}<\% \mathrm{G}$ \\
\hline
\end{tabular}




\begin{tabular}{|c|c|c|c|}
\hline 4 & Janssen et al. ${ }^{28}$ & 2005 & $<\% G$ \\
\hline 5 & Layman et al. ${ }^{29}$ & 2005 & $<\% G$ \\
\hline 6 & Varela et al. ${ }^{30}$ & 2007 & $<\% G$ \\
\hline 7 & Batista et al. $^{31}$ & 2008 & $<\% \mathrm{G}$, \\
\hline 8 & Veloso; Freitas. $^{32}$ & 2008 & $<\% G$ \\
\hline 9 & Macedo; Silva. ${ }^{33}$ & 2009 & $<\% G$ \\
\hline 10 & Rezende et al. ${ }^{34}$ & 2009 & $<\% G$ \\
\hline 11 & Goncalves et al. $^{35}$ & 2010 & $<\% G$ \\
\hline 12 & Wycherley et al. ${ }^{36}$ & 2010 & $<\% G$ \\
\hline 13 & Fisher et al. $^{37}$ & 2011 & $<\% G$ \\
\hline 14 & Monteiro et al. ${ }^{38}$ & 2013 & $<\% G$ \\
\hline 15 & Sanal et al. ${ }^{14}$ & 2013 & $<\% G$ \\
\hline
\end{tabular}

A maioria dos estudos $(n=5)$ trabalhou com 08 exercícios no programa de TF. A Figura 2 demonstra o número de exercícios que cada estudo utilizou, obtendo uma média e desvio padrão $(X \pm D P)$ de $8,8 \pm 1,9$ entre os 15 estudos contemplados. Entre os estudos encontrados 14 deles (93,3\%) obtiveram respostas significativas na redução da porcentagem de gordura $(<\% \mathrm{G})$.

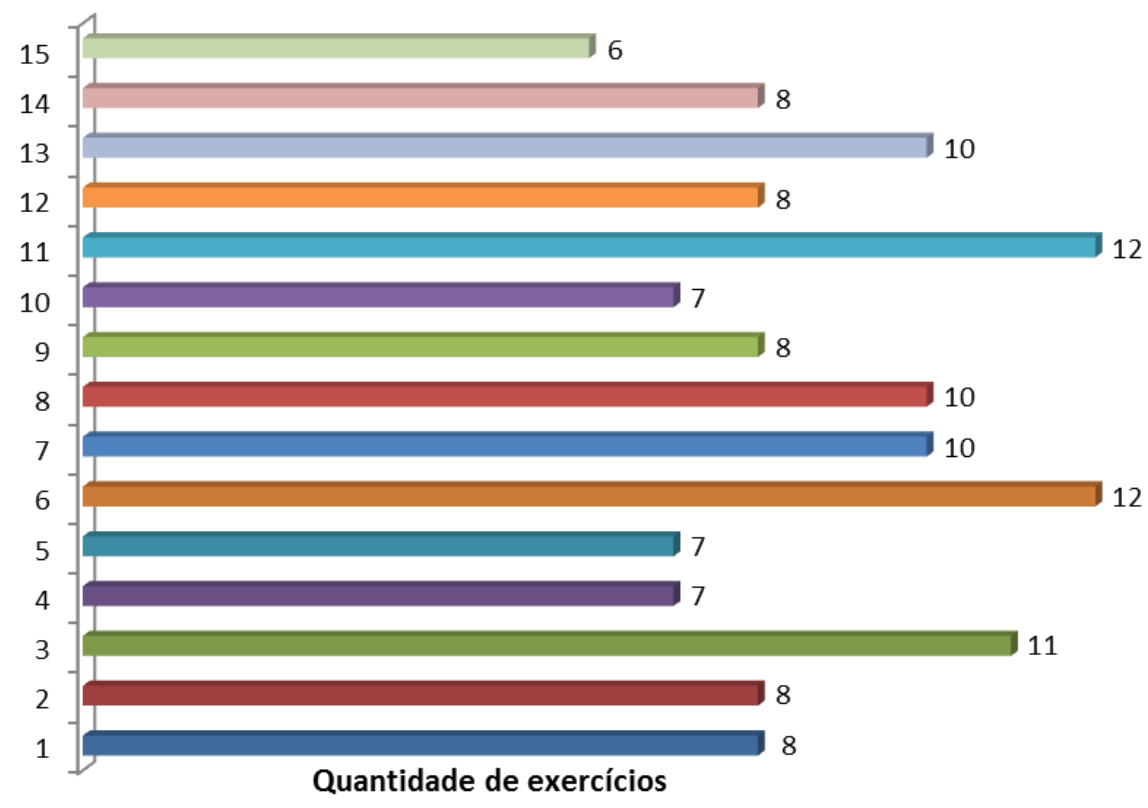

Figura 2. Número de exercícios utilizados nos estudos

\section{DISCUSSÃO}

Tempos atrás, havia pouca sustentação científica para estabelecer que o TF causasse melhoras significativas no tratamento de obesidade ${ }^{39}$, o que atualmente a literatura já demonstra total consenso sobre tal tema, pois, os exercícios intensos, vêm mostrando além de eficientes, superiores aos exercícios 
intensidades menores ${ }^{15,40,41}$. Pois Kang et al. $^{40}$, observaram que exercícios com intensidades de $90 \%$ de $8 \mathrm{RM}$ provocaram maiores níveis de oxidação lipídica quando comparadas a intensidade de ao $60 \%$ de $8 \mathrm{R}$.

Além de o TF gerar emagrecimento, segundo Silva Filho $^{3}$ o indivíduo que realiza TF em um programa de emagrecimento, acaba conseguindo seus objetivos de maneira saudável. Já que nos tratamentos atuais contra obesidade, considera-se indispensável o uso do TF como meio de potencializar a perda de massa gorda e, ao mesmo tempo, preservar a massa magra, corroborando para uma melhor composição corporal total ${ }^{20}$.

Assim como para os demais objetivos, para que o TF provoque o emagrecimento saudável, é necessário que a manipulação das variáveis esteja prescrita de maneira adequada $^{19}$, para que não venha expor os praticantes a situações de riscos ${ }^{23}$. Algumas diretrizes são encontradas na literatura para o uso do TF, porém, quando se fala em número de exercícios, observa-se que não há um consenso estabelecido para que norteie estudos futuros.

Foram encontrados números de exercícios entre $06^{14}$ e $12^{35}$, sendo que quase em todos os estudos as amostras eram de pessoas sedentárias com sobrepeso ou obesidade, com exceção apenas de 02 dos estudos que testaram praticantes de atividade física $^{32,34}$, reafirmando não haver conceito preestabelecido, pois, a maioria dos sujeitos eram sedentários e mesmo assim houve considerável diferença no número de exercícios propostos.

No presente estudo, ao ajustar o número de exercícios em média e desvio padrão, obteve-se o valor de $8,8 \pm 1,9$ e, com exceção de apenas um dos estudos, a maioria $(93,3 \%, n=14)$ provocou redução significativa na porcentagem de gordura. Sendo assim, pressupõe-se que o número de exercícios no TF para gerar emagrecimento aproxima-se de 6 a 12 exercícios por sessão de treinamento.

\section{CONCLUSÃO}

Conclui-se que o número de exercícios utilizados nos ensaios clínicos voltados para o emagrecimento ficou em torno de 6 a 12 exercícios por sessão diária, com número médio de $8,8 \pm 1,9$. Também que, na maioria dos estudos analisados houve redução significativa da porcentagem de gordura.

\section{REFERÊNCIAS}

1. Kraemer WJ, Adams K, Cafarelli E, Dudley GA, Dooly C, Feigenbaum MS, et al. American College of Sports Medicine position stand. Progression models in resistance training for healthy adults. Med Science Sports Exerc. 2002;34(2):364-80. DOI: http://doi.org/10.1097/00005768-200202000$\underline{00027}$

2. Marques Junior NK, Silva Filho JN. Treino de força para o karateca do estilo Shotokan especialista no Kumite. RBPFEX. 2013;7(41):506-33. 
3. Silva Filho JN. Treinamento de força e seus benefícios voltados para um emagrecimento saudável. RBPFEX. 2013;7(40):329-38.

4. World Health Organization. Obesity and Overweight. Globa Strategy. Fact sheet $n^{\circ} 311$ [Internet]. 2013 01.Jan.2014. Available from: http://www.who.int/mediacentre/factsheets /fs311/en/\#

5. World Health Organization. Report of a WHO consultation on obesity. Obesity: preventing and managing the global epidemic Geneva: World Health Organization. 1998.

6. Shaw K, Gennat H, O'Rourke P, Del Mar C. Exercise for overweight or obesity. Cochrane Database Syst Rev. 2006;4. DOI: http://dx.doi.org/10.1002/14651858.CD003817.p $\underline{\mathrm{ub3}}$

7. Epstein LH, Myers MD, Raynor HA, Saelens BE. Treatment of pediatric obesity. Pediatrics. 1998;101(Suppl.2):554-70.

8. Ammon PK. Individualizing the approach to treating obesity. Nurse Pract. 1999;24(2):27-43.

9. Bond Brill J, Perry A, Parker L, Robinson A, Burnett K. Dose-response effect of walking exercise on weight loss. How much is enough? Int J Obes Relat Metabol Disorders. 2002;26(11):1484-93.

DOI: http://dx.doi.org/10.1038/sj.ijo.0802133

10. Mello D, Rosa G, Portela BO, Verdini MLP, Dantas EHM. Efeitos de um programa de caminhada sobre parâmetros biofísicos de mulheres com sobrepeso assistidas pelo Programa de Saúde da Família (PSF). Rev Bras Ativ Fís Saúde. 2012;15(4):224-8.

11. Costa Leite PC, d'Arce FB, Silva CG, Garcia Junior JR. Alteração da gordura corporal e da função cardíaca com exercício físico leve praticado com regularidade. Colloquium Vitae. 2009;1(1):8-16. DOI: http://dx.doi.org/10.5747/cv.2009.v01.n1.v002
12. Marcon ER, Gus I, Neumann CR. Impacto de um programa mínimo de exercícios físicos supervisionados no risco cardiometabólico de pacientes com obesidade mórbida. Arq Bras Endocrinol Metabl. 2011;55(5):331-8. DOI: http://dx.doi.org/10.1590/S0004$\underline{27302011000500006}$

13. Prabhakaran B, Dowling EA, Branch JD, Swain DP, Leutholtz BC. Effect of 14 weeks of resistance training on lipid profile and body fat percentage in premenopausal women. $\mathrm{Br}$ J Sports Med. 1999;33(3):190-5. DOI: http://dx.doi.org/10.1136/bjsm.33.3.190

14. Sanal E, Ardic F, Kirac S. Effects of aerobic or combined aerobic resistance exercise on body composition in overweight and obese adults: gender differences. A randomized intervention study. Eur J Phys Rehab Med. 2013;49(1):1-11.

15. Foureaux G, Pinto KMdC, Dâmaso A. Efeito do consumo excessivo de oxigênio após exercício e da taxa metabólica de repouso no gasto energético. Rev Bras Med Esporte. 2006;12(6):393-8. DOI: http://dx.doi.org/10.1590/S1517$\underline{86922006000600018}$

16. Osterberg KL, Melby CL. Effect of acute resistance exercise on postexercise oxygen consumption and resting metabolic rate in young women. Int J Sport Nutr. 2000;10(1):71-81.

17. Ryan AS, Pratley RE, Elahi D, Goldberg AP. Resistive training increases fat-free mass and maintains RMR despite weight loss in postmenopausal women. J Appl Physiol. 1995;79(3):818-23.

18. Fleck SJ, Kraemer WJ. Fundamentos do treinamento de força muscular. Porto Alegre: Artmed; 2006.

19. Powers SK, Howley ET. Fisiologia do Exercício: Teoria e Aplicação ao 
Condicionamento e ao Desempenho. 6.ed. Barueri: Manole; 2009.

20. Donnelly JE, Blair S, Jakicic J, Manore M, Rankin J, Smith B. American College of Sports Medicine Position Stand. Appropriate physical activity intervention strategies for weight loss and prevention of weight regain for adults. Med Sci Sports Exerc. 2009;41(2):459-71. DOI: http://dx.doi.org/10.1249/MSS.0b013e31819493 $\underline{33}$

21. Rhea MR, Alvar BA, Burkett LN, Ball SD. A meta-analysis to determine the dose response for strength development. Med Sci Sports Exerc. 2003;35(3):456-64. DOI: http://dx.doi.org/10.1249/01.MSS.0000053727.6 3505.D4

22. Silva NL, Farinatti PTV. Influência de variáveis do treinamento contra-resistência sobre a força muscular de idosos: uma revisão sistemática com ênfase nas relações dose-resposta. Rev Bras Med Esporte, 2007, 13.1: 60-6.

DOI:

http://dx.doi.org/10.1590/S1517-

86922007000100014

23. Meeusen $R$, Duclos $M$, Foster C, Fry $A$, Gleeson $M$, Nieman $D$, et al. Prevention, diagnosis, and treatment of the overtraining syndrome: joint consensus statement of the European College of Sport Science and the American College of Sports Medicine. Med Sci Sports Exerc. 2013;45(1):186-205. DOI: http://dx.doi.org/10.1249/MSS.0b013e318279a1 $\underline{0 a}$

24. Thomas JR, Nelson JK, Silverman SJ. Métodos de Pesquisa em Atividade Física. Porto Alegre: Artmed; 2012.

25. Moher D, Liberati A, Tetzlaff J, Altman DG. Preferred reporting items for systematic reviews and meta-analyses: the PRISMA statement. Ann Internal Med. 2009;151(4):264-9. DOI: http://dx.doi.org/10.7326/0003-4819-151-4$\underline{\text { 200908180-00135 }}$
26. Kraemer WJ, Volek JS, Clark KL, Gordon $\mathrm{SE}$, Incledon T, Puhl SM, et al. Physiological adaptations to a weight-loss dietary regimen and exercise programs in women. J Appl Physiol. 1997;83(1):270-9.

27. Dos Santos CF, Crestan TA, Picheth DM, Felix G, Mattanó RS, Porto DB, et al. Efeito de 10 semanas de treinamento com pesos sobre indicadores da composição corporal. Rev Bras Ciên Mov. 2002;10(2):79-84.

28. Janssen I, Fortier A, Hudson R, Ross R. Effects of an energy-restrictive diet with or without exercise on abdominal fat, intermuscular fat, and metabolic risk factors in obese women. Diabetes Care. 2002;25(3):431-8.

DOI: http://dx.doi.org/10.2337/diacare.25.3.431

29. Layman DK, Evans E, Baum JI, Seyler J, Erickson DJ, Boileau RA. Dietary protein and exercise have additive effects on body composition during weight loss in adult women. J Nutr. 2005;135(8):1903-10.

30. Varela AL, Quintans CC, Tranqueira APM, Gasparotto R, da Silva Isaac IA, Estrela RAM, et al. Programa de emagrecimento para mulheres obesas envolvendo variáveis nutricionais, psicológicas e exercício físico. RBONE. 2012;1(6):12-27.

31. Batista FR, de Lira FS, Junior EN, Franchini E. Efeito dos exercícios resistido e aeróbio sobre a massa corporal de mulheres adultas com sobrepeso: Influência da ordem de execução. Aqu Sanny Pesq Saúde. 2008;1(2):109-18.

32. Veloso ALO, Freitas AS. Efeitos crônicos de diferentes estratégias de treinamento de força no processo de emagrecimento em praticantes de musculação. Col Pesq Educ Física. 2008;7(3):157-64.

33. Macêdo D, Silva MS. Efeitos dos programas de exercícios aeróbico e resistido 
na redução de gordura adbominal de mulheres com excesso de peso. Rev Bras Ciên Mov. 2009;17(4):47-54.

34. Rezende FMA, Domiciano TR, de Oliveira Silva DC, Araujo TFV, Gouvêa LF. Efeito de um treinamento resistido periodizado, conforme as fases do ciclo menstrual, na composição corporal e força muscular. Braz J Biomotric. 2009;3(1):65-75.

35. Gonçalves AG, Rodrigues C, Leite RM. O treinamento de força como fator preponderante para perda ponderal em mulheres adultas do município de São José, SC. RBPFEX. 2010;4(22):390-6.

36. Wycherley TP, Noakes M, Clifton PM, Cleanthous X, Keogh JB, Brinkworth GD. A high-protein diet with resistance exercise training improves weight loss and body composition in overweight and obese patients with type 2 diabetes. Diabetes Care. 2010;33(5):969-76.

DOI: http://dx.doi.org/10.2337/dc09-1974

37. Fisher G, Hyatt TC, Hunter GR, Oster RA, Desmond RA, Gower BA. Effect of diet with and without exercise training on markers of inflammation and fat distribution in overweight women. Obesity. 2011;19(6):1131-6. DOI: http://dx.doi.org/10.1038/oby.2010.310

38. Monteiro PA, Antunes BM, Silveira LS, Fernandes RA, Freitas Jr IF. Efeito de um protocolo de treinamento concorrente sobre fatores de risco para o acúmulo de gordura hepática de adolescentes obesos. Medicina (Ribeirao Preto Online). 2013;46(1):17-23.

39. Francischi RP, Pereira LO, Lancha Jr AH. Exercício, comportamento alimentar e obesidade: revisão dos efeitos sobre a composição corporal e parâmetros metabólicos. Rev Paulista Educ Física. 2001;15(2):117-40.
40. Kang J, Rashti SL, Tranchina CP, Ratamess $N A$, Faigenbaum $A D$, Hoffman JR. Effect of preceding resistance exercise on metabolism during subsequent aerobic session. Eur J Appl Physiol. 2009;107.1:43-50. DOI: http://dx.doi.org/10.1007/s00421-009-1100-z

41. Heden, T. et al., One-set resistance training elevates energy expenditure for $72 \mathrm{~h}$ similar to three sets. Eur J Appl Physiol. 2011, 111.3:477-84.

DOI: http://dx.doi.org/10.1007/s00421-010-1666-5

Recebido para publicação em 13/02/2014 Revisado em 26/05/2014

Aceito em 06/06/2014 